\title{
Tunable gene expression system independent of downstream
}

\section{coding sequence}

\section{Supporting Information}

Seung Min Yoo, ${ }^{\dagger}$ Seung-Woon Jung, ${ }^{\dagger}$ Jinho Yeom, ${ }^{\dagger}$ Sang Yup Lee, $,{ }^{\ddagger},{ }^{*}$ and Dokyun $\mathrm{Na}^{*}, \dagger$

†'School of Integrative Engineering, Chung-Ang University, 84 Heukseok-ro, Dongjak-gu, Seoul 06974, Republic of Korea

Department of Chemical and Biomolecular Engineering (BK21 Plus program), KAIST, 291

Daehak-ro, Yuseong-gu, Daejeon 34141, Republic of Korea 


\section{Figures and tables}

Supporting Table 1: The Gibbs free energy of the N-terminal sequence of the genes

\begin{tabular}{lcc}
\hline CDS & N-terminal structure & Gibbs free energy $(\mathrm{kcal} / \mathrm{mol})^{\mathrm{a}}$ \\
\hline$g f p$ & Strong & -20.3 \\
tdTomato & Median & -10.1 \\
$m$ Cherry & Weak & -8.4 \\
$k a n^{\mathrm{R}}-g f p$ & Weak & -9.1 \\
\hline
\end{tabular}

${ }^{a}$ The energies were predicted using ffold $^{*}$. The $\mathrm{N}$-terminal $50 \mathrm{bp}$ of the respective genes were used to predict the energies.

"Rouillard, J.-M., Zuker, M., Gulari, E. 2003. OligoArray 2.0: Thermodynamically improved oligonucleotide design for microarrays. Nucleic Acids Res. 31 (12), 3057-3062. 
Supporting Table 2: Designed spacing nucleotide sequences of the tunable system and their expression levels using $g f p$ and $k a n^{R}$ genes

\begin{tabular}{|c|c|c|c|c|}
\hline \multirow{2}{*}{ Name } & \multirow{2}{*}{ Promoter } & \multirow{2}{*}{ Spacing nucleotide } & \multicolumn{2}{|c|}{ Expression level $^{\mathrm{a}}$} \\
\hline & & & GFP & $\mathrm{Kan}^{\mathrm{R}}$-GFP \\
\hline pA-1 & $\mathrm{P}_{\mathrm{R}}$ & TGAAGCTA & 1.000000 & 1.000000 \\
\hline pA-2 & $\mathrm{P}_{\mathrm{R}}$ & TCCTCGCC & 0.810732 & 0.843819 \\
\hline $\mathrm{pA}-3$ & $\mathrm{P}_{\mathrm{R}}$ & GTTCCCAA & 0.468249 & 0.443933 \\
\hline $\mathrm{pA}-4$ & $\mathrm{P}_{\mathrm{R}}$ & CCATGATA & 0.180343 & 0.267198 \\
\hline $\mathrm{pA}-5$ & $P_{R}$ & CCCCGAA & 0.139491 & 0.214736 \\
\hline pA-6 & $\mathrm{P}_{\mathrm{R}}$ & CCCGGCGC & 0.091051 & 0.111733 \\
\hline $\mathrm{pA}-7$ & $\mathrm{P}_{\mathrm{R}}$ & CGCCCTGC & 0.071357 & 0.104328 \\
\hline pA-8 & $\mathrm{P}_{\mathrm{R}}$ & CCGGCGGG & 0.028182 & 0.066228 \\
\hline pB-1 & BBa_I14032 & TGAAGCTA & \multirow{7}{*}{$\begin{array}{l}0.314138 \\
0.183226 \\
0.063603 \\
0.029170 \\
0.024584 \\
0.032550 \\
0.022823\end{array}$} & \\
\hline pB-2 & BBa_I14032 & TCCTCGCC & & \\
\hline pB-3 & BBa_I14032 & GTTCCCAA & & \\
\hline pB-4 & BBa_I14032 & CCATGATA & & \\
\hline pB-5 & BBa_I14032 & CCCCGAA & & \\
\hline pB-6 & BBa_I14032 & CCCGGCGC & & \\
\hline pB-7 & BBa_I14032 & CGCCCTGC & & \\
\hline pB-8 & BBa_I14032 & CCGGCGGG & 0.009375 & \\
\hline pC-1 & BBa_J23113 & TGAAGCTA & \multirow{7}{*}{$\begin{array}{l}0.007568 \\
0.006378 \\
0.003662 \\
0.001396 \\
0.001084 \\
0.000729 \\
0.000548\end{array}$} & \\
\hline $\mathrm{pC}-2$ & BBa_J23113 & TCCTCGCC & & \\
\hline pC-3 & BBa_J23113 & GTTCCCAA & & \\
\hline pC-4 & BBa_J23113 & CCATGATA & & \\
\hline pC-5 & BBa_J23113 & CCCCGAA & & \\
\hline pC-6 & BBa_J23113 & CCCGGCGC & & \\
\hline pC-7 & BBa_J23113 & CGCCCTGC & & \\
\hline pC-8 & BBa_J23113 & CCGGCGGG & 0.000222 & \\
\hline
\end{tabular}

${ }^{a}$ The leader sequence length used was $288 \mathrm{bp}$. 
Supporting Table 3: E. coli strains and plasmids used in this study

\begin{tabular}{|c|c|c|}
\hline Strain/Plasmid & Description & $\begin{array}{l}\text { Source of } \\
\text { reference }\end{array}$ \\
\hline \multicolumn{3}{|l|}{ Strains } \\
\hline DH5 $\alpha$ & 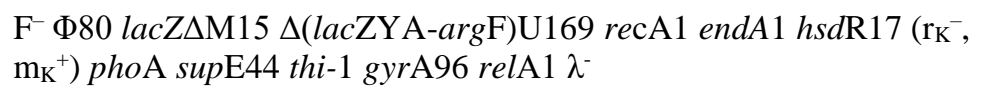 & Invitrogen \\
\hline XQ56 & W3110 $\Delta$ lacI $\Delta$ speE $\Delta$ speG $\Delta$ ygjG $\Delta$ puuPA PdapA::Ptrc & $\begin{array}{c}\text { Qian et al. } \\
\text { (2011) }\end{array}$ \\
\hline NMH26 & $\begin{array}{l}\text { W3110 } \Delta \text { lacI } \Delta \text { speE } \Delta \text { speG } \Delta \operatorname{argI} \Delta \text { puuPA } \Delta \text { putAP } \Delta \text { proP } \Delta \text { speC- } \\
\text { potE } \Delta \text { speF PargECBH::Ptrc PspeF-potE::Ptrc PargD::Ptrc }\end{array}$ & $\begin{array}{l}\text { Noh et al. } \\
\text { (2017) }\end{array}$ \\
\hline DP09 & $\begin{array}{l}\text { W3110 } \\
\text { thrAC1034T lysCC1055T Pppc::Ptrc PaspC::Ptrc } \Delta p f k A\end{array}$ & $\begin{array}{l}\text { Chae et al. } \\
\text { (2015) }\end{array}$ \\
\hline $\mathrm{PRO} 2$ & 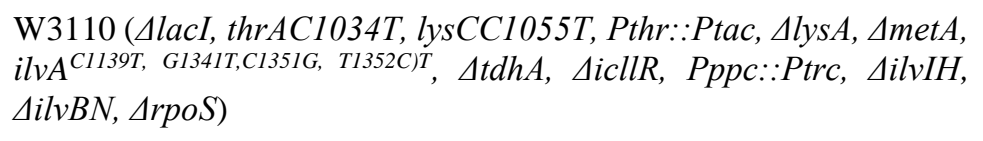 & $\begin{array}{l}\text { Choi et al. } \\
\text { (2012) }\end{array}$ \\
\hline \multicolumn{3}{|l|}{ Plasmids } \\
\hline pWA & APR, ColE1 ori from pBR322, mutiple cloning site, $2.561 \mathrm{~kb}$ & \\
\hline $\mathrm{p} 15 \mathrm{~K}$ & $\mathrm{KmR}, \mathrm{p} 15 \mathrm{~A}$ ori, mutiple cloning site, $3.025 \mathrm{~kb}$ & \\
\hline p15CadA & $\begin{array}{l}\text { KmR, E. coli cadA gene in EcoRI-SacI site of pTac } 15 \mathrm{~K}, 5 \mathrm{nt} \\
\text { (GCGTC) inserted upstream of the EcoRI-cadA-SacI cassette, } 6.1 \mathrm{~kb}\end{array}$ & $\begin{array}{l}\text { Qian et al. } \\
\quad(2011)\end{array}$ \\
\hline p15PP3533 & $\begin{array}{l}\mathrm{Km}^{\mathrm{R}}, P . \text { putida } \mathrm{PP} 3533 \text { gene in SacI-XbaI site of pTac } 15 \mathrm{~K}, \mathrm{p} 15 \mathrm{~A} \text { ori, } \\
5.0-\mathrm{kb}\end{array}$ & $\begin{array}{l}\text { Noh et al. } \\
\text { (2017) }\end{array}$ \\
\hline $\begin{array}{l}\text { p15DDoptpasp } \\
\text { C }\end{array}$ & $\begin{array}{l}\text { pTac15k derivative, containing dat- } d d c \text { genes from } A \text {. } \\
\text { baumannii }(6.8 \mathrm{~kb}) \text { containing tac promoter in front of aspC gene } \\
(8.1 \mathrm{~kb})\end{array}$ & $\begin{array}{l}\text { Chae et al. } \\
\text { (2015) }\end{array}$ \\
\hline $\begin{array}{l}\text { pBRthrABC_pt } \\
\text { ac_cimA_ptac_- } \\
\text { ackA }\end{array}$ & $\begin{array}{l}\mathrm{Ap}^{\mathrm{R}} \text { tac promoter, } M . \quad \text { jannaschii } \quad \text { cimA and ackA cloned into } \\
\text { pBRthrABC }\end{array}$ & $\begin{array}{l}\text { Choi et al. } \\
\text { (2012) }\end{array}$ \\
\hline $\begin{array}{l}\text { pTacDA_ptac } \\
\text { adhE }\end{array}$ & $\mathrm{Km}^{\mathrm{R}}$, tac promoter, $a d h E^{m u t}$ cloned into pTacDA & $\begin{array}{l}\text { Choi et al. } \\
\text { (2012) }\end{array}$ \\
\hline
\end{tabular}


Supporting Table 4: Oligonucleotides used in this study

\begin{tabular}{|c|c|c|c|}
\hline Constructs & Template & Primer name & Sequence $\left(5^{\prime}-3^{\prime}\right)$ \\
\hline $\begin{array}{l}\text { PR Promoter } \\
(\mathrm{BBa}-\mathrm{R} 0051)\end{array}$ & None & PR-F & $\begin{array}{l}\text { TAACACCGTGCGTGTTGACTATTTTACCTCTGGC } \\
\text { GGTGATAATGGTTGC }\end{array}$ \\
\hline I14032 & None & & $\begin{array}{l}\text { AATTACAGAGCTCTGGTGCAAAACCTTTCGCGG } \\
\text { TATGGCATGATAGCGCCAAGCACATATCGAAAG } \\
\text { GATAGTC }\end{array}$ \\
\hline $\mathbf{J} 23113$ & None & & $\begin{array}{l}\text { AATTACAGAGCTCCTGATGGCTAGCTCAGTCCTA } \\
\text { GGGATTATGCTAGCAAGCACATATCGAAAGGAT } \\
\text { AGTC }\end{array}$ \\
\hline \multirow[t]{2}{*}{ lacI } & \multirow{2}{*}{$\begin{array}{l}\text { E. coli } \\
\text { W3110 } \\
\text { DNA }\end{array}$} & LacI_F_BamHI & $\begin{array}{l}\text { AATAAGGATCCATGAAACCAGTAACGTTATACG } \\
\text { ATGTCG }\end{array}$ \\
\hline & & LacI_R_XbaI & AATAAGTCTAGATTACTGCCCGCTTTCCAGTCGG \\
\hline \multirow[t]{2}{*}{$c I$} & $\begin{array}{l}\text { Phage } \lambda \\
\text { DNA }\end{array}$ & cI_F_PacI & $\begin{array}{l}\text { AATGTATTAATTAAAAGGAGACCCGGGATATGA } \\
\text { GCAC }\end{array}$ \\
\hline & & cI_R_PacI & $\begin{array}{l}\text { AATGTATTAATTAATTAGCCAAACGTCTCTTCAG } \\
\text { GCCA }\end{array}$ \\
\hline \multirow[t]{2}{*}{$\operatorname{cadA}$} & p15CadA & cadA_F_BamHI & $\begin{array}{l}\text { AATAAGGATCCATGAACGTTATTGCAATATTGA } \\
\text { ATCACATGG }\end{array}$ \\
\hline & & cadA_R2_XbaI & $\begin{array}{l}\text { AATAAGTCTAGAGGGTACCGAGCTCTTATTTTTT } \\
\text { GCTT }\end{array}$ \\
\hline \multirow[t]{2}{*}{ PP3533 } & p15PP3533 & $\begin{array}{l}\text { PP3533_F_Bam } \\
\text { HI }\end{array}$ & $\begin{array}{l}\text { AATGTAGGATCCATGACGTATTTCATTGATGTTC } \\
\text { CAACCATG }\end{array}$ \\
\hline & & PP3533_R_XbaI & $\begin{array}{l}\text { AATGTATCTAGATTATTAGGCAACCCGTCGGATA } \\
\text { CG }\end{array}$ \\
\hline \multirow[t]{2}{*}{ atoDA } & $\begin{array}{l}\text { pTacDA_pt } \\
\text { ac_adhE }{ }^{\text {mut }}\end{array}$ & atoDA_F_SacI & $\begin{array}{l}\text { AATGTAGAGCTCATGAAAACAAAATTGATGACA } \\
\text { TTACAAGACGCCACC }\end{array}$ \\
\hline & & atoDA_R_XbaI & $\begin{array}{l}\text { AATGTATCTAGATCATAAATCACCCCGTTGCG } \\
\text { TATTCAG }\end{array}$ \\
\hline \multirow[t]{2}{*}{$a d h E^{m u t}$} & $\begin{array}{l}\text { pTacDA_pt } \\
\text { ac_adhE }\end{array}$ & $\begin{array}{l}\text { adhEmut_F_Ba } \\
\text { mHI }\end{array}$ & $\begin{array}{l}\text { AATGTAGGATCCATGGCTGTTACTAATGTCGCTG } \\
\text { AAC }\end{array}$ \\
\hline & & $\begin{array}{l}\text { adhEmut_R_Xb } \\
\text { aI }\end{array}$ & $\begin{array}{l}\text { AATGTATCTAGATTAAGCGGATTTTTTCGCTTT } \\
\text { TTTCTCAGC }\end{array}$ \\
\hline \multirow[t]{2}{*}{$\operatorname{aspC}$} & $\begin{array}{l}\text { p15DDoptp } \\
\text { aspC }\end{array}$ & aspC_F_BamHI & $\begin{array}{l}\text { AATGTAGGATCCATGTTTGAGAACATTACCGCC } \\
\text { GC }\end{array}$ \\
\hline & & aspC_R_XbaI & $\begin{array}{l}\text { AATGTATCTAGATTACAGCACTGCCACAATCGCT } \\
\text { TC }\end{array}$ \\
\hline
\end{tabular}


A
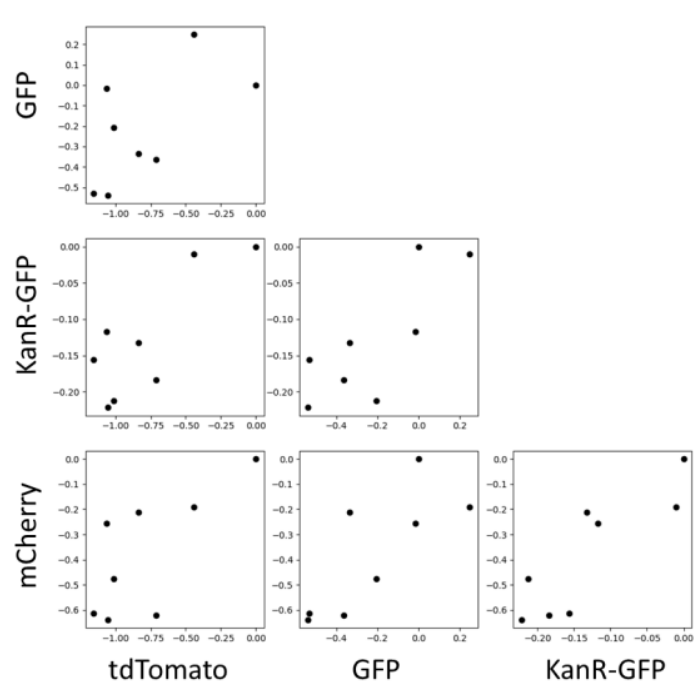

C
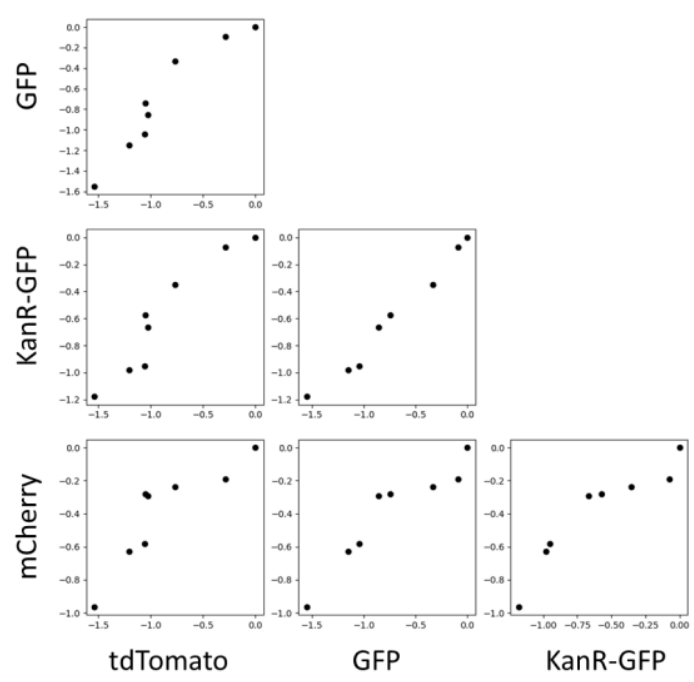

B
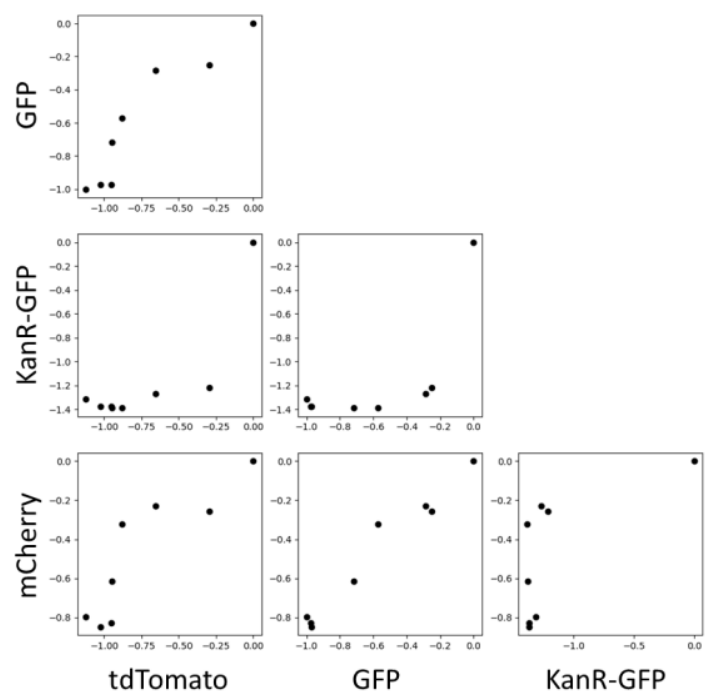

D
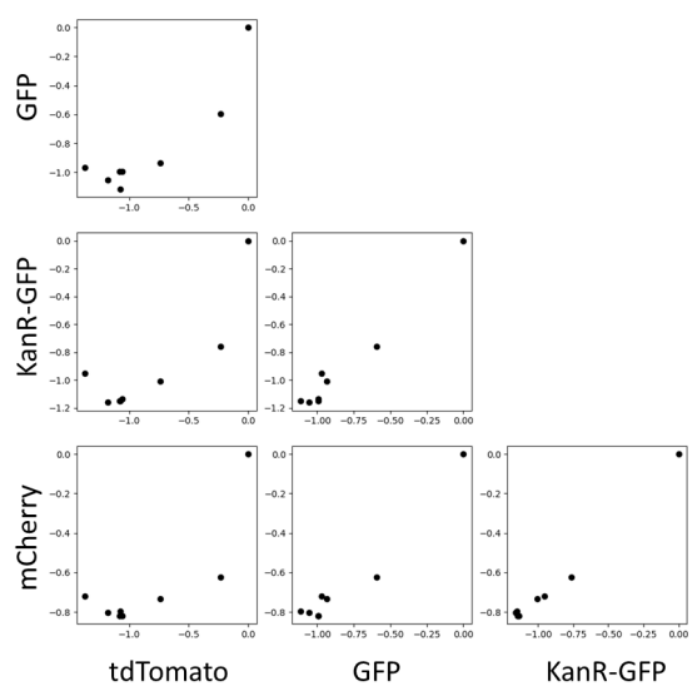

Supporting Figure 1. Pairwise expression comparison of four different proteins with respect

4 to the length of the leader sequence. The length of the leader sequence was 99bp (A), 198bp

5 (B), 288bp (C), and 399bp (D). The expression levels were normalized and then converted to 6 a log scale.

7 
Promoter region

TTAATTAA AAGGAG NNNNNNNN $\frac{\text { Spacer }}{\text { SD }}$ - $\begin{gathered}\text { SD \& } \\ \text { spacer }\end{gathered}$

ATG AAA ATT GCG GCC CTG AAA CAG AAA ATC

$\begin{array}{llllllllll}\mathbf{M} & \mathrm{K} & \mathrm{I} & \mathbf{A} & \mathbf{A} & \mathrm{L} & \mathrm{K} & \mathbf{Q} & \mathrm{K} & \mathrm{I}\end{array}$

GCG AGC CTG AAA CAG GGG GCT GCG GCA GGC

$\begin{array}{llllllllll}\mathbf{A} & \mathbf{S} & \mathrm{L} & \mathrm{K} & \mathbf{Q} & \mathbf{G} & \mathbf{A} & \mathbf{A} & \mathbf{A} & \mathbf{G}\end{array}$

GCC CAT CAC CAC CAC CAT CAT CAT CAC GGT

\begin{tabular}{l|llllllll|l|} 
A & H & H & H & H & H & H & H & H & G
\end{tabular}

GCA GGG GCA GGC GCT GGT GCG GGT GCC GGA

$\begin{array}{llllllllll}\text { A } & \text { G } & \text { A } & \text { G } & \text { A } & \text { G } & \text { A } & \text { G } & \text { A } & \text { G }\end{array}$

TCT CTG TTC AAC AAA GAA CAA CAA AAT GCT

$\begin{array}{llllllllll}\mathbf{S} & \mathbf{L} & \mathbf{F} & \mathbf{N} & \mathrm{K} & \mathbf{E} & \mathbf{Q} & \mathbf{Q} & \mathbf{N} & \mathbf{A}\end{array}$

TTC TAT GAA ATT TTA CAT TTA CCT AAC TTA

Leader

sequence

$\begin{array}{llclllllll}\mathbf{F} & \mathbf{Y} & \mathbf{E} & \mathbf{I} & \mathbf{L} & \mathbf{H} & \mathbf{L} & \mathbf{P} & \mathbf{N} & \mathbf{L}\end{array}$

ACT GAA GAA CAA CGT AAC GGC TTC ATC CAA

AGC CTT AAA GAC GAT CCT TCA GTG AGC AAA

$\begin{array}{llllllllll}\mathbf{S} & \mathbf{L} & \mathbf{K} & \mathrm{D} & \mathbf{D} & \mathbf{P} & \mathbf{S} & \mathbf{V} & \mathbf{S} & \mathbf{K}\end{array}$

GAA ATT TTA GCA GAA GCT AAA AAG CTA AAT

$\begin{array}{llllllllll}\mathbf{E} & \mathbf{I} & \mathbf{L} & \mathbf{A} & \mathbf{E} & \mathbf{A} & \mathbf{K} & \mathbf{K} & \mathbf{L} & \mathbf{N}\end{array}$

GAT GCA CAA GCA GCC GCA GGA TCC TAC AAG 7

$\left.\begin{array}{cccccccccc}\mathbf{D} & \mathbf{A} & \mathbf{Q} & \mathbf{A} & \mathbf{A} & \mathbf{A} & \mathbf{G} & \mathbf{S} & \mathbf{Y} & \mathbf{K} \\ \text { GAA } & \text { TTC } & \text { GGT } & \text { GCA } & \text { GGG } & \text { GCA } & \text { GGC } & \text { GCT } & \text { GGT } & \text { GCG }\end{array}\right]$ (BamHI/EcoRI)

$\begin{array}{llllllllll}\mathbf{E} & \mathbf{F} & \mathbf{G} & \mathbf{A} & \mathbf{G} & \mathbf{A} & \mathbf{G} & \mathbf{A} & \mathbf{G} & \mathbf{A}\end{array}$

GGT GCC GAA ATC GCA GCC CTG GAG TAT GAA

$\begin{array}{llllllllll}\mathbf{G} & \mathbf{A} & \mathbf{E} & \mathbf{I} & \mathbf{A} & \mathbf{A} & \mathbf{L} & \mathbf{E} & \mathbf{Y} & \mathbf{E}\end{array}$

ATT GCG GCG CTG GAA CAG TAA TAA

$\begin{array}{llllllll}\text { I } & \mathbf{A} & \mathbf{A} & \mathrm{L} & \mathbf{E} & \mathrm{Q} & * & *\end{array}$

$\downarrow$ Gly-Ala linker

His-tag

B domain

13 Supporting Figure 2. Designed leader sequence. Nucleotide sequences of the system gene,

14 including the leader sequence. Pink, Gly-Ala linker; dark blue, His-tag region; yellow, B 15 domain region. The detailed leader sequences of 99bp, 198bp, 288bp, and 399bp are found in

\section{Supporting Data 1-4.}



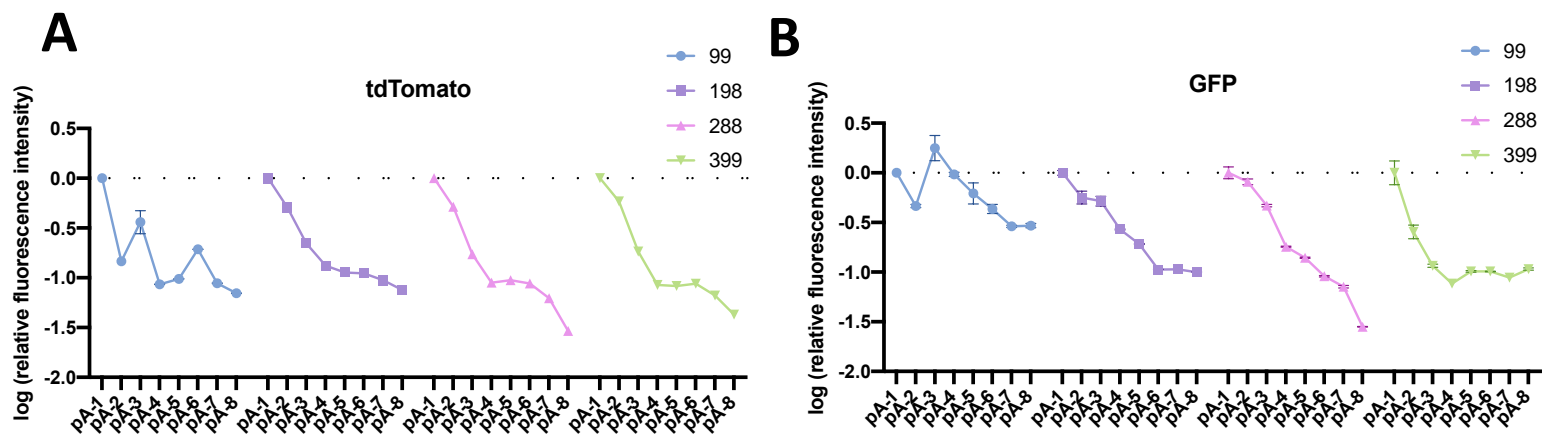

C
D

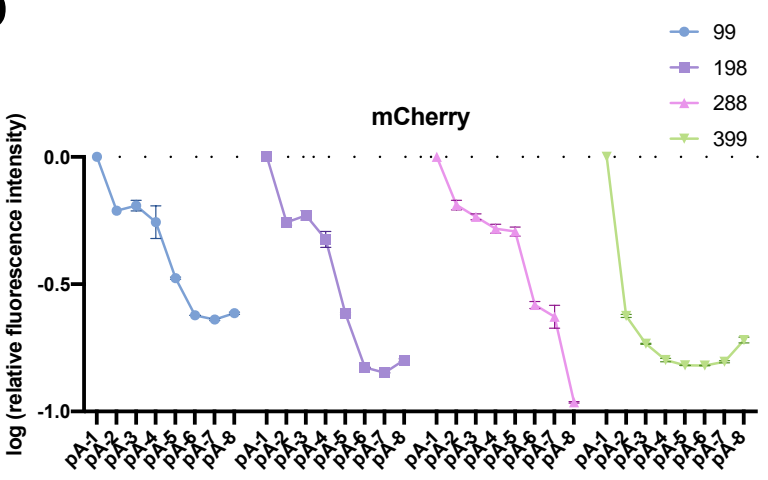

4 Supporting Figure 3. Evaluation of the effect of leader sequence length on the expression

5 levels of four genes. The tdTomato (A), GFP (B), $\operatorname{Kan}^{\mathrm{R}}$-GFP (C), and mCherry (D). 
A

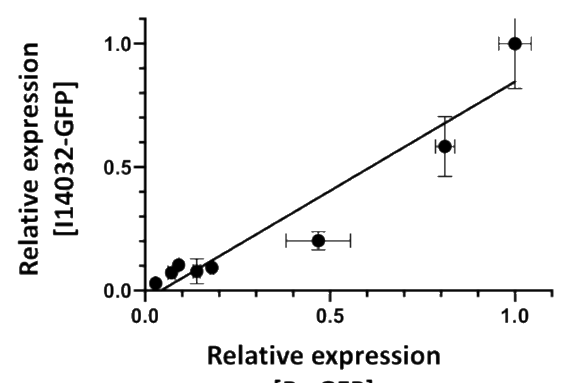

B [PR-GFP]

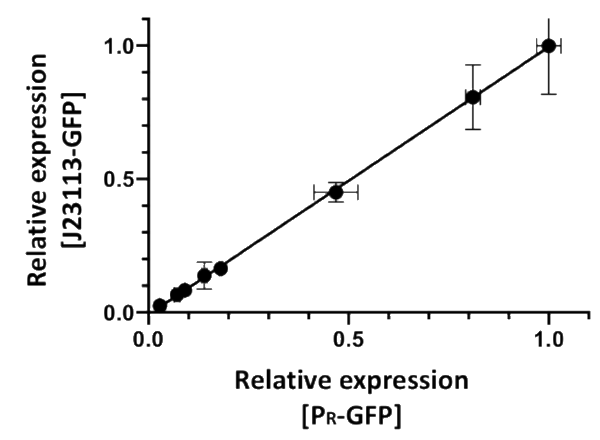

C

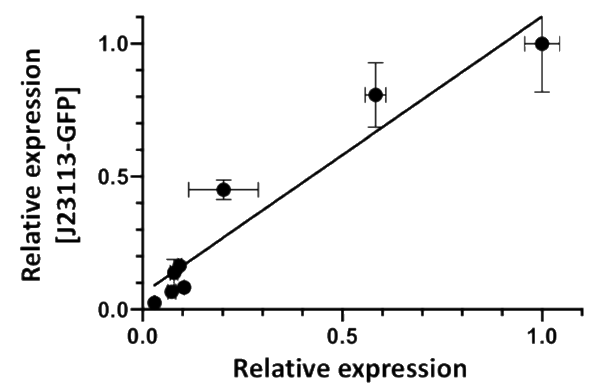

[114032-GFP]

32 Supporting Figure 4. Relative expression levels of GFP proteins generated by combinations 33 of 8 spacers and 3 promoters. The efficacies of combinations of 8 spacers and 3 promoters 34 were analyzed by measuring fluorescence intensities of GFP. (A) $P_{R}-G F P$ versus I14032-GFP. 35 (B) $P_{R}-G F P$ versus J23113-GFP. (C) J23113-GFP versus I14032-GFP. Mean values \pm SD from 36 duplicate experiments are shown. 

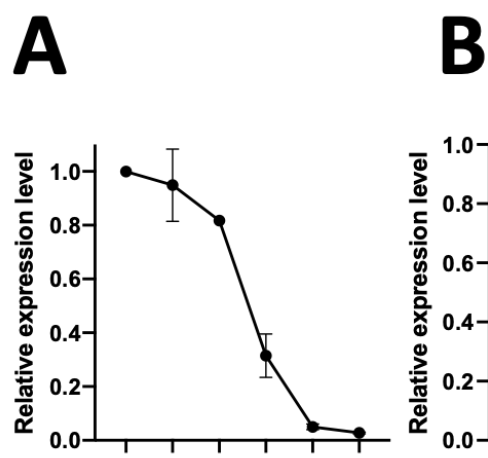

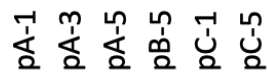
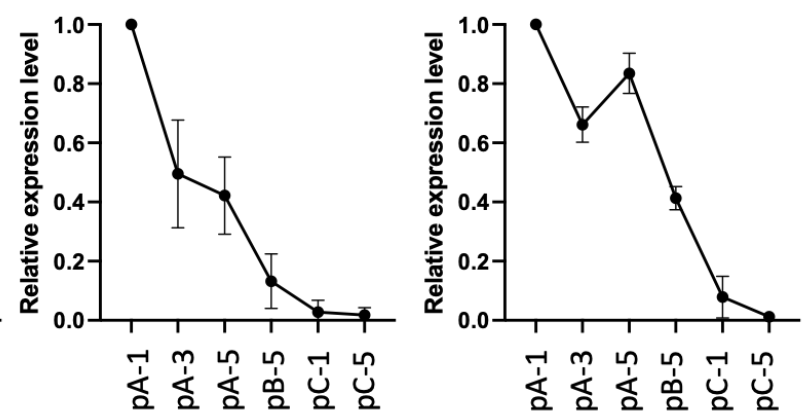

42

43 Supporting Figure 5. Quantitation of the expressed proteins of CadA, PP3533, and AdhE ${ }^{\text {mut }}$.

44 The expression levels of three different proteins were estimated by measuring the intensities of

45 Western-blot bands; (A) CadA, (B) PP3533, and (C) AdhE ${ }^{\text {mut }}$. The measured intensities were 46 normalized to band intensities of the gel stained.

47

48

49 


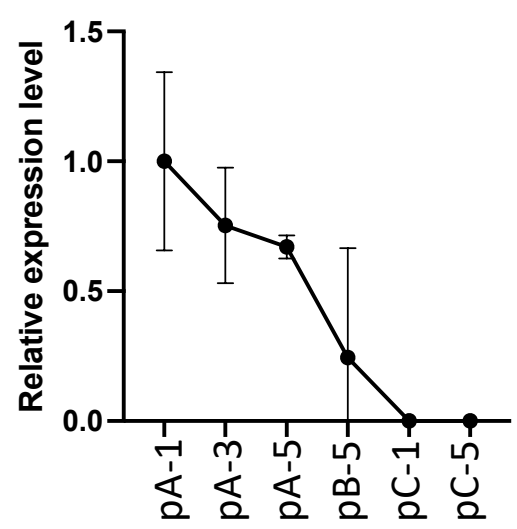

52

Supporting Figure 6. Quantitation of the expressed $\mathrm{AdhE}^{\mathrm{mut}}$ in cell-free system. The

54 expression level of $\mathrm{AdhE}^{\text {mut }}$ was estimated by measuring the intensities of Western-blot

55 bands. Standard error was calculated from three replicates. 